\title{
Cerebrospinal fluid amyloid beta and glial fibrillary acidic protein concentrations in Huntington's disease
}

Sara Korpela $^{1 *}$, Jimmy Sundblom ${ }^{2}$, Henrik Zetterberg ${ }^{3,4,5,6,7}$, Radu Constantinescu ${ }^{8}$, Per Svenningsson ${ }^{9}$, Martin Paucar ${ }^{9}$ Valter Niemelä ${ }^{10 *}$,

1 Department of Medicine, Neurology, Västerås Central Hospital, Västerås, Sweden 2 Institute for Neuroscience, Neurosurgery, Uppsala University, Uppsala, Sweden 3 Department of Psychiatry and Neurochemistry, Institute of Neuroscience and Physiology, the Sahlgrenska Academy at the University of Gothenburg, Mölndal, Sweden 4 Clinical Neurochemistry Laboratory, Sahlgrenska University Hospital, Mölndal, Sweden

5 Department of Neurodegenerative Disease, UCL Institute of Neurology, Queen Square, London, UK

6 UK Dementia Research Institute at UCL, London, UK

7 Hong Kong Center for Neurodegenerative Diseases, Hong Kong, China

8 Institute of Neuroscience and Physiology, Clinical Neuroscience, The Sahlgrenska Academy, University of Gothenburg, Gothenburg, Sweden

9 Department of Clinical Neuroscience, Karolinska Institutet, Stockholm, Sweden

10 Institute for Neuroscience, Neurology, Uppsala University, Uppsala, Sweden

* sara.sisko.susanna.korpela@regionvastmanland.se *valter.niemela@neuro.uu.se

\section{Abstract}

\section{Introduction}

Huntington's disease (HD) is a genetic incurable lethal disease. Biomarkers are needed for objective assessment of disease progression. Evidence supports both complex protein aggregation and astrocyte activation in HD. This study assesses the 42 amino acid long amyloid beta (A $\beta 42)$ and glial fibrillary acidic protein (GFAP) as potential biomarkers in the cerebrospinal fluid (CSF) of HD mutation carriers.

\section{Methods}

CSF was obtained from manifest HD patients (ManHD), premanifest HD-gene-expansion carriers (PreHD) and gene-negative controls (controls). Disease Burden Score (DBS) and Total Functional Capacity (TFC) were calculated. Protein concentrations were measured by enzyme-linked immunosorbent assays (ELISA) and intergroup differences were analysed using Mann-Whitney U test. Spearman correlations were calculated to assess disease stage association. Age-adjustment was included in the statistical tests.

\section{Results}

The study enrolled $27 \mathrm{ManHD}$ and 13 PreHD subjects. The number of controls differed in the analysis of $\mathrm{A} \beta 42$ and GFAP $(\mathrm{n}=19$, and 8 respectively). A $\beta 42$ levels were higher in ManHD (mean $741 \mathrm{ng} / \mathrm{l}$, SD 361) compared with PreHD (mean 468 ng/l, SD 184) (p 
$=0.025$ ). Likewise GFAP concentration was higher in ManHD (mean $435 \mathrm{ng} / \mathrm{l}$, SD 255) compared with both PreHD (mean $266 \mathrm{ng} / \mathrm{l}$, SD 92.4) $(\mathrm{p}=0.040)$ and controls (mean $208 \mathrm{ng} / \mathrm{l}, \mathrm{SD} 83.7)(\mathrm{p}=0.011)$. GFAP correlated with DBS $(\mathrm{r}=0.361, \mathrm{p}=0.028)$, TFC $(\mathrm{r}=-0.463, \mathrm{p}=0.005)$, and 5-year risk of onset in PreHD $(\mathrm{r}=0.694, \mathrm{p}=0.008)$. In contrast, there was no correlation between A $\beta 42$ concentration and DBS, TFC or 5-year risk of onset.

\section{Conclusion}

CSF A 342 levels did not correlate with disease stage suggesting no A 3 aggregation in HD. GFAP is a potential biomarker in HD with association to disease stage. Validation in larger HD cohorts and potential correlations with clinical phenotype would be of interest.

\section{Introduction}

Huntington's disease (HD) is an autosomal dominant neurodegenerative disorder caused by pathological expansions in the polyglutamine (CAG expansion) region in exone 1 in the huntingtin gene (HTT) on chromosome 4q [1]. CAG encodes for the aminoacid glutamine $(\mathrm{Q})$ and the CAG expansion leads to the formation of a protein with an abnormal poly-Q tail. Mutated HTT (mHTT) interacts with other proteins, accumulates and causes dysfunction and eventually degeneration and death of the neurons [2]. These changes have shown to be most prominent in capsula interna and striatum [3]. Symptoms of HD are progressive and include movement disorders, psychiatric symptoms [4] and cognitive decline [5]. There is no disease-modifying drug (DMD) or curative treatment available for HD so current treatment options are purely symptomatic. Several promising gene-based clinical trials are under way. HTT lowering therapies hold the promise of a disease modifiying therapy for HD. The diagnosis of HD is based on the presence of symptoms, clinical signs and confirmed by genetic testing. In the era of emerging therapies there is a need for biofluid markers to assess the progression of HD and thereby evaluate the utility of DMDs objectively. Some promising candidate biomarkers have recently been studied [6], of which neurofilament light protein (NfL), a broad biomarker for neurodegenerative diseases and neuroinflammation such as multple sclerosis (MS), seems to be the most promising with association to both disease onset and stage in HD [7-9], as well as mHTT [10]. Total tau (an axonal microtubule-stabilizing protein), another biomarker for neurodegeneration, has also been associated with the severity of symptoms, especially psychiatric symptoms but the data is less convincing compared with NfL $[7,9,11-14]$. Here we wanted to extend the CSF analysis of neurodegenerative markers with studies on Amyloid $\beta(\mathrm{A} \beta)$ protein and glial fibrillary acidic protein (GFAP) which have not been reported in HD before.

Dysregulation of amyloid $\beta(A \beta)$ protein causes extracellular aggregation of the 42 amino acid form of $A \beta(A \beta 42)$ in brain tissue. $A \beta 42$ is considered to be the most pathogenic form of $A \beta[15]$. A $\beta 42$ aggregation leads to neuronal dysfunction mediated by excitotoxicity and neuroinflammation [16-18] and cereral hypoperfusion [19]. One form of A ppathology is cerebral amyloid angiopathy (CAA), where the amyloid accumulates in the vascular walls [20]. A $\beta 42$ aggregation is a hallmark of Alzheimer's disease (AD) $[21,22]$, where CSF A $\beta 42$ levels are reduced [23-26]. This decrease is likely due to increased amyloid deposition in the brain and decreased clearance into the CSF [27], and there is an inverse correlation with higher whole brain amyloid deposition load and low CSF A $\beta 42[26,28,29]$. Low A $\beta 42$ levels may also be seen following axonal injury caused by e.g. traumatic brain injury [30], and in primary neurodegenerative disorders, such as dementia with Lewy bodies (DLB) [31] and Parkinson's disease 
dementia [32]. CSF A 342 has been examined as a biomarker in depression where higher brain Aßburden in PiB-PET was found to be associated with increasing anxious-depressive symptoms over time in cognitively normal older individuals [33]. Studies on MS have found correlation between lower CSF A $\beta 42$ levels and disease progression [34], including cognitive impairment [35], and higher gadolinium-enhancing lesion burden on magnetic resonance imaging [35].

GFAP (glial fibrillary acidic protein) is an intermediary fibrillary protein in the glial cells (astrocytes) and increased GFAP immunoreactivity is associated with gliosis and slowly progressing neuronal damage $[36,37]$. It is used as a diagnostic marker in gliomas, especially astrocytomas [38,39], is released in blood following ischaemic stroke [40], and is a marker for inflammation and disease stage in, e.g. MS [41-43]. Highly increased CSF GFAP concentration is seen in neuromyelitis optica spectrum disorders (NMOSD) due to rapid astrocytic damage $[44,45]$. A post-mortem study performed on HD patients revealed astrocytosis in moderate, but not the earliest pathological stages of HD [46]. Similarly, trials on the HD mouse model R6/2 have shown increased GFAP levels in astrocytes of moderate and late disease stages. [47].

This study aims to investigate alterations of A $\beta 42$ and GFAP levels as potential biomarkers for disease onset and progression in HD.

\section{Materials and methods}

\section{Definition of participants and clinical assessment}

The participants were recruited from the HD clinic at Uppsala University Hospital, from Karolinska University Hospital in Stockholm and Sahlgrenska University Hospital, Gothenburg, and were either premanifest gene-expansion carriers or manifest HD subjects. The gene-negative controls were recruited for the study amongst healthy individuals without signs of neurological disease. Premanifest gene-expansion carriers were defined as individuals with CAG expansion (over 35 repeats) of the HD-gene (HTT) and with a diagnostic confidence level (DCL) below 4. Manifest HD subjects were defined as individuals with a CAG-expansion (over 35 repeats) in the HD-gene and a DCL of 4. Disease burden score (DBS) was calculated for each HD patient using the formula (CAG repeat length-35.5) $\mathrm{x}$ age to estimate the cumulative HD pathology exposure. Total functional capacity (TFC) and corresponding disease stages (1-4) were assessed. The study was conducted in accordance with the declaration of Helsinki and was approved by the regional ethical review board in Uppsala, Sweden (DNR 2012/274) All participants signed an informed consent before study entry.

\section{CSF sample collection and handling}

CSF was collected by lumbar puncture according to standardized protocol for procedure, materials and handling, but the time of the lumbar puncture and relation to meals varied. Polypropylene tubes and collecting vessels were used to avoid protein adsorption. The CSF was put on ice and centrifuged at 4 degrees Celsius and $1300 \mathrm{G}$ for 10 minutes. The acellular proportion was pipetted off for storage at minus 70 degrees Celsius until the time of analysis.

\section{Biochemical analyses}

CSF A 342 concentration was measured by INNOTEST enzyme-linked immunosorbent assay (ELISA) (Fujurebio, Ghent, Belgium). CSF GFAP concentration was measured using an in-house ELISA, as previously described in detail [48]. 


\section{Statistical analyses}

Tests for normality of distribution included Shapiro-Wilk and inspection of histogram and the skewness statistics were applied. Age was approximately normally distributed, but the protein levels were not. Intergroup differences in protein levels were tested with non-parametric tests (Mann-Whitney U test). If there was deemed to be an association to age and/sex this variable was included as a covariant in a linear regression analysis model. We performed Spearman rank correlation with all gene-expansion carriers pooled into one group to assess the correlation between the protein levels and TFC as well as DBS. All statistical analyses were performed on cross-sectionally sampled data. The level of significance was defined by p-value less than 0.05. Statistical analyses were performed using SPSS Statistics Subscription Build 1.0.0.1461

\section{Results}

The study enrolled 59 participants (mean age; standard deviation, range see Table 1 and Table 2).

CSF A 342 concentration was measured in 59 participants (mean age 49.2; SD 16.1, range $20-78$ years). 13 of these $(22.0 \%)$ were premanifest gene-expansion carriers, 27 (45.8\%) manifest HD patients and $19(32.2 \%)$ gene-negative controls.

CSF GFAP concentration was measured in 48 participants (mean age 48.9, SD 16.2, range $20-78$ years). 13 of these $(27.1 \%)$ were premanifest gene-expansion carriers, 27 $(56.3 \%)$ manifest HD patients and $8(16.7 \%)$ gene-negative controls. The characteristics of these subgroups are described in Table 1 and Table 2.

The protein levels were also compared to those of the laboratorys age-stratified reference rates. The group sizes differ because of insufficient amounts of CSF from several individuals, precluding quantitative analysis of both A $\beta 42$ and GFAP.

Table 1. Characteristics of the study population; $A \beta 42$

Age, CAG repeat length and Disease burden are presented as Mean (SD)

\begin{tabular}{|l|l|l|l|l|l|}
\hline Group & N & Age & Male:Female ratio & CAG & Disease burden \\
\hline Total & 59 & $49.2(16.1)$ & $29: 30$ & - & - \\
\hline Controls & 19 & $48.5(16.8)$ & $7: 12$ & - & - \\
\hline Premanifest HD & 13 & $37.2(11.5)$ & $7: 6$ & $43.6(3.9)$ & $274.6(75.8)$ \\
\hline Manifest HD & 27 & $55.4(14.4)$ & $15: 12$ & $43.5(3.3)$ & $419.6(187.7)$ \\
\hline
\end{tabular}

Table 2. Characteristics of the study population; GFAP

Age, CAG repeat length and Disease Burden are presented as Mean (SD)

\begin{tabular}{|l|l|l|l|l|l|}
\hline Group & N & Age & Male:Female ratio & CAG & Disease burden \\
\hline Total & 48 & $48.9(16.2)$ & $28: 20$ & - & - \\
\hline Controls & 8 & $42.9(18.5)$ & $5: 3$ & - & - \\
\hline Premanifest HD & 13 & $37.2(11.5)$ & $7: 6$ & $43.6(3.9)$ & $274.6(75.8)$ \\
\hline Manifest HD & 27 & $56.3(13.6)$ & $16: 11$ & $43.6(3.2)$ & $403.4(202.9)$ \\
\hline
\end{tabular}

\section{CSF A $\beta 42$ and GFAP concentration differences between groups}

There was no association between age and CSF A 342 concentration $(\mathrm{r}=-0.322 \mathrm{p}=$ 
but regarding GFAP a significant positive correlation between age and protein concentration has been noted in healthy individuals [48].

Regarding $\mathrm{A} \beta 42$, the premanifest and the manifest HD group differed significantly in age $(\mathrm{p}=0.001)$, and regarding GFAP the manifest HD group was significantly older than both the premanifest HD group and control group $(\mathrm{p}=0.0003$ and $\mathrm{p}=0.043$ respectively), due to the natural course of the disease. To exclude potential confounding age was included as a covariate in the statistical analyses.

Figure 1 shows the concentrations of both proteins in the three groups. CSF A $\beta 42$ concentration was significantly higher in the manifest HD group (mean $741 \mathrm{ng} / \mathrm{l}$, SD 361) compared with the premanifest HD group (mean $468 \mathrm{ng} / \mathrm{l}$, SD 183, ( $\mathrm{p}=0.025)$. The difference remained significant after adjustment for age $(\mathrm{p}=0.03)$. There were no significant differences between the gene-negative controls (mean $535 \mathrm{ng} / \mathrm{l}$, SD 238) and the manifest HD group, but a trend towards higher levels in the manifest HD group was noted $(\mathrm{p}=0.076$, age-adjusted $\mathrm{p}=0.112)$. There were no significant differences between gene-negative controls and premanifest gene-expansion carriers $(\mathrm{p}=0.454$, age-adjusted $\mathrm{p}=0.626$ ).

Fig 1. Concentrations of $A \beta 42$ and GFAP by group.

Protein concentrations plotted un-adjusted by group, p-values are adjusted for age. Boxes show first and third quartiles, the central bands show the median, and the whiskers show data within 1.5 IQR of the median. (A) A $\beta 42$ levels were higher in the manifest group compared with the premanifest gene-expansion carriers. (B) GFAP levels were higher in the manifest group compared with both the premanifest gene-expansion carriers and healthy controls.

CSF GFAP concentration was significantly higher in the manifest HD group (mean $435 \mathrm{ng} / \mathrm{l}$, SD 255) compared with both the premanifest HD group (mean $266 \mathrm{ng} / \mathrm{l}, \mathrm{SD}$ 92.4) $(\mathrm{p}=0.040)$ and the gene-negative controls (mean $208 \mathrm{ng} / \mathrm{l}, \mathrm{SD} 83.7)(\mathrm{p}=0.011)$. These differences remained significant even after adjustment for age $(\mathrm{p}=0.018, \mathrm{p}=$ 0.043 , respectively). CSF GFAP concentration did not differ significantly between the premanifest group and the gene-negative controls $(\mathrm{p}=0.070$, age-adjusted $\mathrm{p}=0.313)$.

\section{Associations of CSF A $\beta 42$ and GFAP concentrations with disease progression}

As shown in Figure 2, we found no correlation between CSF A $\beta 42$ concentration and DBS $(r=0.207, p=0.220)$. Neither was there any correlation between A $\beta 42$ concentration and TFC $(\mathrm{r}=-0.270, \mathrm{p}=0.111)$. After adjustment for age the correlation between $\mathrm{A} \beta 42$ concentration and TFC became statistically significant $(\mathrm{p}=$ $0.024)$. A $\beta 42$ concentration did not correlate with 5-year risk of onset among premanifest gene-expansion carriers $(\mathrm{r}=0.218, \mathrm{p}=0.474)$.

\section{Fig 2. Correlations between protein levels and disease progression in} Huntington's disease.

A 342 concentration does not correlate with (A) disease burden, (C) total functional capacity or (E) 5-year risk of onset, whereas GFAP concentration correlates with (B) Disease burden, (D) total functional capacity and (F) most strongly with 5-year risk of onset.

CSF GFAP concentration correlated positively with DBS and inversely to TFC with a significant correlation $(\mathrm{r}=0.361, \mathrm{p}=0.028$ and $\mathrm{r}=-0.463, \mathrm{p}=0.005$, respectively). Correlation between protein levels and TFC remained statistically significant even after adjustment for age $(\mathrm{p}=0.001)$. DBS was not adjusted for age since this variable is 
already included in the composite score. GFAP levels correlated with 5-year risk of onset among premanifest gene-expansion carriers $(\mathrm{r}=0.694, \mathrm{p}=0.008)$.

We did not find any correlation between CSF GFAP and A $\beta 42$ concentrations ( $\mathrm{r}=-$ 0.097, $\mathrm{p}=0.522$ ).

\section{Discussion}

In this exploratory CSF study we found significantly increased CSF A $\beta 42$ concentration in the manifest HD group compared with the premanifest gene-expansion carriers. There was a non-significant tendency to lower levels of A 342 in the premanifest HD group compared with the healthy controls. However, A $\beta 42$ concentration did not correlate with Disease Burden Score, Total Functional Capacity, or 5-year risk of onset.

A recent study demonstrated an association between lower levels of CSF amyloid precursor protein (APP) and worse clinical phenotype and lower cognitive performance in HD patients [52], the strongest relationship observed with composite UHDRS score. APP is a transmembrane protein with multiple physiological functions, including regulating brain iron homeostasis [53], and it is cleaved by beta- and gamma-secretase to form $\mathrm{A} \beta$ peptides $[54,55]$. Our previous finding of decreased CSF transthyretin in $\mathrm{HD}$ patients [56] has also been reported in $\mathrm{AD}$, where it possibly contributes to the failure of cerebral amyloid clearance [57]. This might allude to similarly decreased A $\beta 42$ levels in HD, as noted in several other neurodegenerative disorders, most notably in $\mathrm{AD}$ [24-29]. On the contrary, we found the highest levels of A $\beta 42$ in the manifest HD group. The mechanism underlying this finding is unknown. Nevertheless, the data corroborate that $\mathrm{A} \beta$ aggregation is not a feature of $\mathrm{HD}$ [58].

The present study found higher CSF GFAP concentration in the manifest HD group compared with both premanifest gene-expansion carriers and healthy controls. There was a non-significant trend towards higher CSF GFAP concentration in the premanifest group compared with controls. Further, GFAP concentration correlated positively with Disease Burden score and inversely with the Total Functional Capacity in the pooled gene-expansion carrier group. The strongest correlation was found between GFAP levels and 5-year risk of onset among the premanifest gene-expansion carriers. As GFAP is a marker of astrogliosis and degenerative process [36,37], as well as a known marker for neuroinflammation $[36,37,41-43]$, and/or astrocyte damage $[44,45]$, the interpretation of elevated GFAP levels is not so straightforward as to what kind of underlying pathological processes might be involved. The levels of GFAP also tend to rise with ageing in healthy individuals, most probably as a sign of astroglial filament formation in the CNS [48]. Still, our findings are in line with pathology studies of HD patients that have described astrocytosis in moderate, but not the earliest pathological grades of HD in humans [46], as well as findings in the R6/2 mouse, where GFAP was elevated in astrocytes of moderate and late disease stages as a sign of classical astrocyte activation [47]. Previous CSF studies in HD patients have shown increased CSF levels of YKL-40, another astrocytic activation marker, as a late feature of HD [9,59], and a correlation to several markers of neurodegeneration [59]. YKL-40 is secreted by astrocytes and is increased in many inflammatory CNS disorders [60]. Evidence from CSF studies in HD mainly supports activation of the innate immune system of the CNS This may reflect inflammation caused by neurodegeneration in the later stages of the disease [9]. However, an early increase of IL- 6 and IL-8 levels in both CSF and plasma suggest an innate immune response both centrally and peripherally in HD [61]. There is also evidence of T-cell mediated inflammation ahead of disease onset [59]. The tendency to elevation of GFAP in premanifest gene-expansion carriers together with a strong correlation with risk of onset suggests astrocyte activation predating phenoconversion. Some of the premanifest gene-expansion carriers were far from predicted disease onset 
and this could be a reason that GFAP was not clearly elevated in this group.

To the best of our knowledge, this is the first CSF study performed on humans to assess the role of $\mathrm{A} \beta$ and GFAP as potential biomarkers in HD. However astrocyte activation in the form of astrocytosis and neuroinflammation has previously been linked to the pathophysiology of HD, especially in the late disease stages so this is in line with the finding of elevated GFAP levels amongst the manifest HD patients. Part of the elevation might be due to the normal aging process, as the manifest HD patients are usually older compared to the premanifest HD-gene-expansion carriers, but the difference remained significant even after taking this fact into account. The finding of the highest $A \beta 42$ levels in the manifest HD group was unexpected considering that in other neurodegenerative diseases, A $\beta$ tends to decrease in CSF along with the level of neurodegeneration in parallel with worse clinical outcome. Limitations in this study include the exploratory nature and a small sample size. Different ages between groups was of concern and the fact that the groups differed in size. The medications used by the patients and their potential effect on the protein levels was not assessed.

Nonetheless, we believe that these findings may be of relevance regarding the involvement of astrocyte activation, neuroinflammation and gliosis in HD. GFAP may have a role in assessing the severity of HD, and could potentially also serve as a surrogate end-point in clinical trials. Before taking these findings into clinical practice there is a need for validation in a larger HD cohort and assessment of correlations with clinical phenotype would be of interest.

\section{Supporting Information}

S1 Table. Dataset including clinical characteristics of study participants and protein concentrations. (PDF)

\section{Acknowledgments}

We want to thank the participants of this study for donating their samples

\section{Author Contributions}

\section{Planning:}

VN, JS

Conceptualization:

VN, JS, RC, PS, MP

Data collection:

VN, JS, RC, PS, MP, SK, HZ

Formal analysis:

HZ, SK

Funding acquisition:

JS, VN, HZ, PS

Investigation:

VN, HZ, SK

Methodology:

VN, SK, JS, PS

Project administration:

VN, RC, PS, JS, SK

Software:

SK, VN 


\author{
Visualization: \\ VN, SK \\ Writing - original draft: \\ SK, VN, JS \\ Writing - review \& editing: SK, VN, JS, HZ, RC, PS, MP
}

\title{
References
}

1. The Huntington's Disease Collaborative Research Group. A novel gene containing a trinucleotide repeat that is expanded and unstable on Huntington's disease chromosomes. Cell 1993;72:971-983

2. Ross CA, Aylward EH, Wild EJ, Langbehn DR, Long JD, Warner JH, et al. Huntington's disease: natural history, biomarkers and prospect for therapeutics. Nature reviews Neurology 2014; 10(4):204-16.

3. Nopoulos PC. Huntington disease: a single-gene degenerative disorder of the striatum. Dialogues in Clinical Neuroscience. 2016; 18(1):91-8.

4. Paoli RA, Botturi A, Ciammola A, Silani V, Prunas C, Lucchiari C, et al. Neuropsychiatric Burden in Huntington's disease. Brain Sci. 2017; 7(6)

5. Baake V, Reijntjes R, Dumas EM, Thompson JC, Network RIotEHsD, Roos RAC. Cognitive decline in Huntington's disease expansion gene carriers. Cortex. 2017; 95:51-62.

6. Byrne LM, Wild EJ. Cerebrospinal Fluid Biomarkers for Huntington's Disease. Journal of Huntington's disease. 2016; 5(1): 1-13.

7. Niemelä V, Landtblom A-M, Blennow K, Sundblom J. Tau or neurofilament light - Which is the more suitable biomarker for Huntington's disease? PLoS ONE 2017; 12(2):e0172762.doi:10.1371/journal.pone.0172762

8. Constantinescu R, Romer M, Oakes D, Rosengren L, Kieburtz K. Levels of the light subunit of neurofilament triplet protein in cerebrospinal fluid in Huntington's disease. Parkinsonism \& related disorders. 2009; 15(3):245-8

9. Vinther-Jensen T, Bornsen L, Budtz-Jorgensen E, Ammitzboll C, Larsen IU, Hjermind LE, et al. Selected CSF biomarkers indicate no evidence of early neuroinflammation in Huntington's disease. Neurology ßneuroimmunology \& neuroinflammation. 2016;3(6):e287.

10. Wild EJ, Boggio R, Langbehn D, Robertson N, Haider S, Miller JR, et al. Quantification of mutant huntingtin protein in cerebrospinal fluid from Huntington's disease patients. The Journal of clinical investigation. 2015;125(5): 1979-86

11. Spillantini MG, Goedert M. Tau pathology and neurodegeneration. The Lancet Neurology. 2013; 12(6):609-22

12. Gratuze M, Cisbani G, Cicchetti F, Planel E. Is Huntington's disease a tauopathy? Brain: a journal of neurology. 2016; 139(Pt4):1014-25

13. Constantinescu R, Romer M, Zetterberg H, Rosengren L, Kieburtz K. Increased levels of total tau protein in the cerebrospinal fluid in Huntington's disease.

Parkinsonism \& related disorders. 2011; 17(9):714-5 
14. Rodrigues FB, Byrne L, McColgan P, Robertson N, Tabrizi SJ, Leavitt BR, et al. Cerebrospinal fluid total tau concentration predicts clinical phenotype in Huntington's disease. Journal of neurochemistry. 2016. Epub 2016/06/28

15. Duering M, Grimm MOW, Grimm HS, Schröde J, Hartmann T. Mean age of onset in familial Alzheimer's disease is determined by amyloid beta 42. Neurobiol Aging. 2005;26:785-8.

16. Johnstone M, Gearing AJ, Miller KM. A central role for astrocytes in the inflammatory response to beta-amyloid: chemokines, cytokines and reactive oxygen species are produced. J Neuroimmunol 1999;93:182-193.

17. Fiala M, Zhang L, Gan X, Sherry B, Taub D, Graves MC,et al. Amyloid-beta induces chemokine secretion and monocyte migration across a human blood-brain barrier model. Mol Med 1998;4:480-489.

18. Neuroinflammatory Working Group. Inflammation and Alzheimer's disease. Neurobiol Aging 2001;21:383-421.

19. Thomas T, Thomas G, McLendon C, Sutton T, Mullan M. BetaAmyloid-mediated vasoactivity and vascular endothelial damage. Nature 1996;380:168-171.

20. Vinters HV. Cerebral amyloid angiopathy. A critical review. Stroke. 1987;18:311-324

21. Findeis MA. The role of amyloid beta-peptide 42 in Alzheimer's disease. Pharmacol Ther 2007;116:266-286

22. Blennow K, Hampel H, Weiner M, Zetterberg H. Cerebrospinal fluid and plasma biomarkers in Alzheimer disease. Nat Rev Neurol. 2010; 6(3):131-44. https://doi.org/10.1038/nrneurol.2010.4 PMID: 20157306

23. Hampel H, Teipel SJ, Fuchsberger T, Andreasen N, Wiltfang J, Otto M, et al. Value of CSF $\beta$-amyloid 1-42 and tau as predictors of Alzheimer's disease in patients with mild cognitive impairment. Mol Psychiatry. 2004; 9(7):705-10. https://doi.org/10.1038/sj.mp.4001473 PMID: 14699432

24. Moonis M, Swearer JM, Dayaw MPE, St. George-Hyslop P, Rogaeva E, Kawarai T, et al. Familial Alzheimer disease: Decreases in CSF A $\beta 42$ levels precede cognitive decline. Neurology. 2005; 65(2):323-5. https://doi.org/10.1212/01.wnl.0000171397.32851.bc PMID: 16043812

25. Sunderland T, Linker G, Mirza N, Putnam KT, Friedman DL, Kimmel LH, et al. Decreased $\beta$-Amyloid1-42 and Increased Tau Levels in Cerebrospinal Fluid of Patients with Alzheimer Disease. J Am MedAssoc. 2003; 289(16):2094-103.

26. Grimmer T, Riemenschneider M, Förstl H, Henriksen G, Klunk WE, Mathis CA, et al. Beta Amyloid in Alzheimer's Disease: Increased Deposition in Brain Is Reflected in Reduced Concentration in Cerebrospinal Fluid. Biol Psychiatry.2009;65(11):927-34.https://doi.org/10.1016/j.biopsych.2009.01.027PMID: 19268916

27. Weller RO. How well does the CSF inform upon pathology in the brain in Creutzfeldt-Jakob and Alzheimer's diseases? J Pathol. 2001; 194(1):1-3. https://doi.org/10.1002/1096-9896(200105)194:1;1::AID-PATH871¿3.0.CO;2-M PMID: 11329133. 
28. Seppälä TT, Nerg O, Koivisto AM, Rummukainen J, Puli L, Zetterberg H, et al. CSF biomarkers for Alzheimer disease correlate with cortical brain biopsy findings. Neurology 2012;78(20):1568-75.https://doi.org/10.1212/WNL.0b013e3182563bd0PMID: 22517093

29. Palmqvist S, Zetterberg H, Mattsson N, Johansson P, Minthon L, Blennow K, et al. Detailed comparison of amyloid PET and CSF biomarkers for identifying early Alzheimer disease. Neurology 2015;85(14):1240-9. https://doi.org/10.1212/WNL.0000000000001991 PMID: 26354982

30. Franz G, Beer R, Kampfl A, Engelhardt K, Schmutzhard E, Ulmer H, Deisenhammer F. Amyloid beta 1-42 and tau in cerebrospinal fluid after severe traumatic brain injury. Neurology 2003;60(9):1457-61. doi: 10.1212/01.wnl.0000063313.57292.00. PMID: 12743231.

31. Abdelnour C, van Steenoven I, Londos E, Blanc F, Auestad B, Kramberger MG, et al.; European DLB Consortium. Alzheimer's disease cerebrospinal fluid biomarkers predict cognitive decline in lewy body dementia. Movement Disorders 2016;3(8):1203-08. https://doi.org/10.1002/mds.26668

32. Siderowf A, Xie SX, Hurtig H, et al. CSF amyloid $\beta 1-42$ predicts cognitive decline in Parkinson disease. Neurology 2010;75:1055-1061.

33. Donovan NJ, Locascio JJ, Marshall GA, Gatchel J, Hanseeuw BJ,Rentz DM, et al.; Harvard Aging Brain Study. Longitudinal association of Amyloid beta and anxious-depressive symptoms in cognitively normal older adults. Am J Psychiatry 2018; 175:530-537; doi: 10.1176/appi.ajp.2017.17040442

34. Pietroboni AM, Caprioli M, Carandini T, Scarioni M, Ghezzi L, Arighi A, et al. CSF $\beta$-amyloid predicts prognosis in patients with multiple sclerosis. Mult Scler. 2019;25(9):1223-1231. doi: 10.1177/1352458518791709.

35. Mori F, Rossi S, Sancesario G, et al. Cognitive and Cortical Plasticity Deficits Correlate with Altered Amyloid- $\beta$ CSF Levels in Multiple Sclerosis. Neuropsychopharmacol 2011;36:559-568.https://doi.org/10.1038/npp.2010.187

36. Finch CE. Neurons, glia, and plasticity in normal brain aging. Neurobiol Aging. 2003;24 Suppl 1:S123-7; discussion S131. doi: 10.1016/s0197-4580(03)00051-4. PMID: 12829120.

37. Hausmann ON. Post-traumatic inflammation following spinal cord injury. Spinal Cord. 2003;41(7):369-78. doi: 10.1038/sj.sc.3101483. PMID: 12815368.

38. van Bodegraven EJ, van Asperen JV, Robe PAJ, Hol EM. Importance of GFAP isoform-specific analyses in astrocytoma. Glia 2019; 67(8): 1417-1433

39. Tichy J, Spechtmeyer S, Mittelbronn M, Hattingen E, Rieger J, Senft C, Foerch C. Prospective evaluation of serum glial fibrillary acidic protein (GFAP) as a diagnostic marker for glioblastoma. J Neurooncol 2016; 126(2): 361-9.

40. Liu G, Geng J. Glial fibrillary acidic protein as a prognostic marker of acute ischemic stroke. Hum Exp Toxicol 2018; 37(10): 1048-1053

41. Abdelhak A, Huss A, Kassubek J, Tumani H, Otto M. Serum GFAP as a biomarker for disease severity in multiple sclerosis. Sci Rep 2018; 8(1): 14798 
42. Martinez, M. A. et al. Glial and neuronal markers in cerebrospinal fluid predict progression in multiple sclerosis. Multiple sclerosis 2015;21:550-561, https://doi.org/10.1177/1352458514549397

43. Kassubek R, Gorges M, Schocke M, Hagenston VAM, Huss A, Ludolph AC, et al. GFAP in early multiple sclerosis: A biomarker for inflammation. Neurosci Lett. 2017 Sep 14;657:166-170. doi: 10.1016/j.neulet.2017.07.050. Epub 2017 Aug 9. PMID: 28802830.

44. Takano R, Misu T, Takahashi T, Sato S, Fujihara K, Itoyama Y. Astrocytic damage is far more severe than demyelination in NMO. Neurology 2010; 75(3): 208-216. DOI: https://doi.org/10.1212/WNL.0b013e3181e2414b

45. Uzawa A, Mori M, Sawai S, Masuda S,Muto M, Uchida T, et al. Cerebrospinal fluid interleukin-6 and glial fibrillary acidic protein levels are increased during initial neuromyelitis optica attacks. Clinica Chimica Acta 2013;421:181-183,ISSN00098981,https://doi.org/10.1016/j.cca.2013.03.020.(https://www.sciencedirect.com/science/ar

46. Myers RH, Vonsattel JP Fau, Paskevich PA, Kiely DK, Stevens TJ, Cupples LA, Richardson EP Jr., et al. Decreased neuronal and increased oigodendroglial densities in Huntingtons disease caudate nucleus. J Neuropathol Exp Neurol. 1991; 50(6)(0022-3069(Print)): 729-42.PMID:1836225.

47. Tong X, Ao Y, Faas GC, Nwaobi SE, Xu J, Haustein MD, A, et al. Astrocyte Kir4.1 ion channel deficits contribute to neuronal dysfunction in Huntington's disease model mice. Nat Neurosci. 2014 May;17(5):694-703. doi: 10.1038/nn.3691. Epub 2014 Mar 30. PMID: 24686787; PMCID: PMC4064471.

48. Rosengren LE, Wikkels $\varnothing$ C, Hagberg L, A sensitive ELISA for glial fibrillary acidic protein: application in CSF of adults. Journal of Neuroscience Methods 1994; 41(2):197-204.

49. Taghdiri, F., Gumus, M., Algarni, M. et al. Association Between Cerebrospinal Fluid Biomarkers and Age-related Brain Changes in Patients with Normal Pressure Hydrocephalus. Sci Rep 2020;10:9106. https://doi.org/10.1038/s41598-020-66154-y

50. Andreasen N, Hesse C, Davidsson P, Minthon L, Wallin A, Winblad B, et al. Cerebrospinal fluid beta-amyloid(1-42) in Alzheimer disease: differences between early- and late-onset Alzheimer disease and stability during the course of disease. Arch Neurol. 1999; 56(6):673-80. doi: 10.1001/archneur.56.6.673. PMID: 10369305.

51. Sjögren M, Vanderstichele H, Agren H, Zachrisson O, Edsbagge M, Wikkelsø C, et al. Tau and Abeta42 in cerebrospinal fluid from healthy adults 21-93 years of age: establishment of reference values. Clin Chem. 2001 Oct;47(10):1776-81. PMID: 11568086 .

52. Lowe AJ, Sjödin S, Rodrigues FB, Byrne LM, Blennow K, Tortelli R, et al. Cerebrospinal fluid endo-lysosomal proteins as potential biomarkers for Huntington's disease. PLoS ONE 2020;15(8):e0233820. https://doi.org/10.1371/journal.pone.0233820

53. Duce JA, Tsatsanis A, Cater MA, James SA, Robb E, Wikhe K, et al. Iron Export Ferroxidase Activity of B-Amyloid Precursor Protein is Inhibited by Zinc in Alzheimer's Disease. Cell 2010; 142(6): 857-67. https://doi.org/10.1016/j.cell.2010.08.014 
54. Müller UC, Zheng H. Physiological functions of APP family proteins. Cold Spring Harb Perspect Med.2012; 2(2):1-18.

55. Haass C, Kaether C, Thinakaran G, Sisodia S. Trafficking and proteolytic processing of APP. ColdSpring Harb Perspect Med. 2012; 2(5):1-25.

56. Niemela V, Landtblom AM, Nyholm D, Kneider M, Constantinescu R, Paucar M, et al. Proenkephalin Decreases in Cerebrospinal Fluid with Symptom Progression of Huntington's Disease. Mov Disord. 2021 Feb;36(2):481-491. doi: 10.1002/mds.28391. Epub 2020 Nov 28. PMID: 33247616; PMCID: PMC7984171.

57. Li X, Buxbaum JN. Travsthyretin and the brain re-visited: Is neuronal synthesis of transthyretin protective in Alzheimers disease? Molecular Neurodegeneration 2011; 6(79). https://doi.org/10.1186/1750-1326-6-79

58. St-Amour, I., Turgeon, A., Goupil, C. et al. Co-occurrence of mixed proteinopathies in late-stage Huntington's disease. Acta Neuropathol 135, 249-265 (2018). https://doi.org/10.1007/s00401-017-1786-7

59. Niemelä V, Burman J, Blennow K, Zetterberg H, Larsson A, Sundblom J. Cerebrospinal fluid sCD27 levels indicate active T cell-mediated inflammation in premanifest Huntington's disease. PLoS ONE 2018;13(2):e0193492.

https://doi.org/10.1371/journal.pone.0193492

60. Bonneh-Barkay D, Wang G, Starkey A, Hamilton RL, Wiley CA. In vivo CHI3L1 (YKL-40) expression in astrocytes in acute and chronic neurological diseases. JNeuroinflammation 2010; 7:34. https://doi.org/10.1186/1742-2094-7-34 PMID:20540736

61. Björkqvist M, Wild EJ, Thiele J, Silvestroni A, Andre R, Lahiri N, et al. A novel pathogenic pathway of immune activation detectable before clinical onset in Huntington's disease. J Exp Med. 2008 Aug 4;205(8):1869-77. doi: 10.1084/jem.20080178. Epub 2008 Jul 14. PMID: 18625748; PMCID: PMC2525598. 


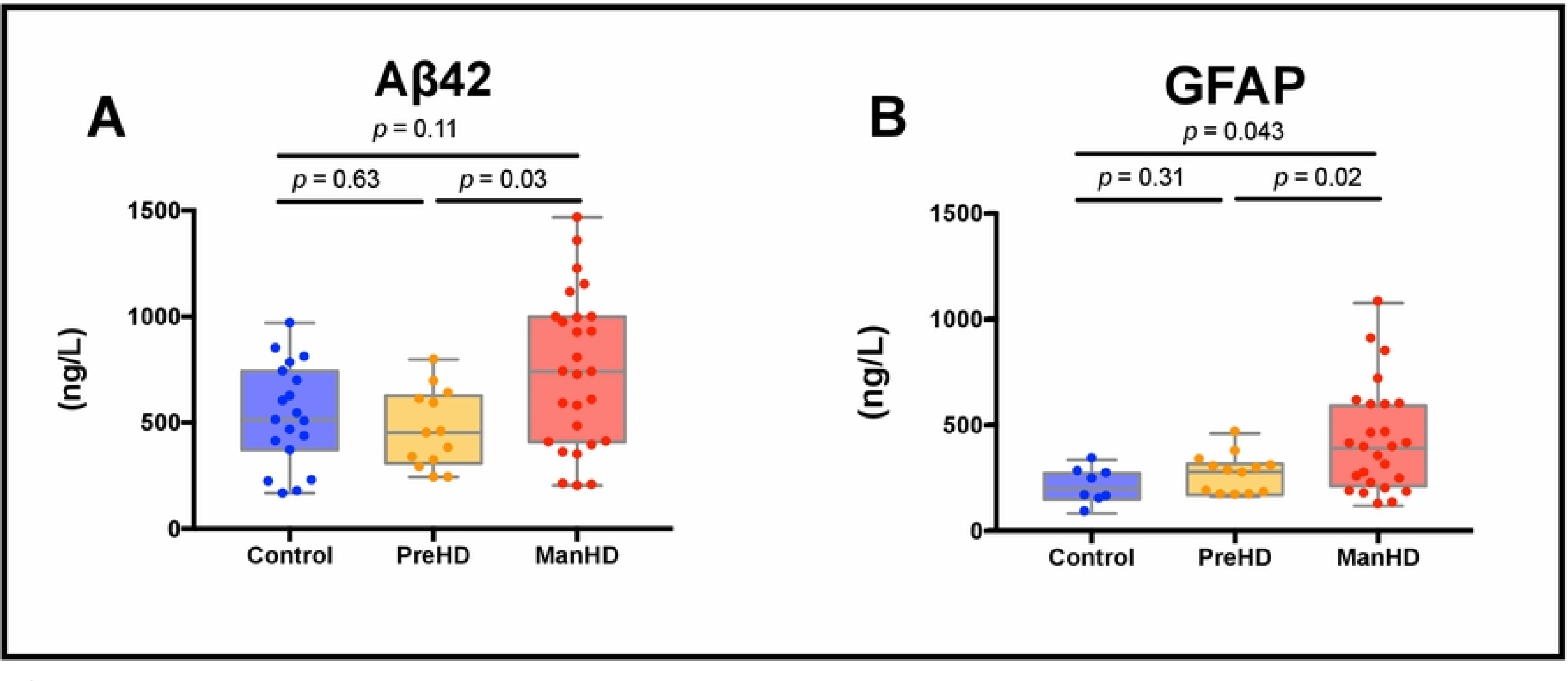

Figure 1 
A 342

A
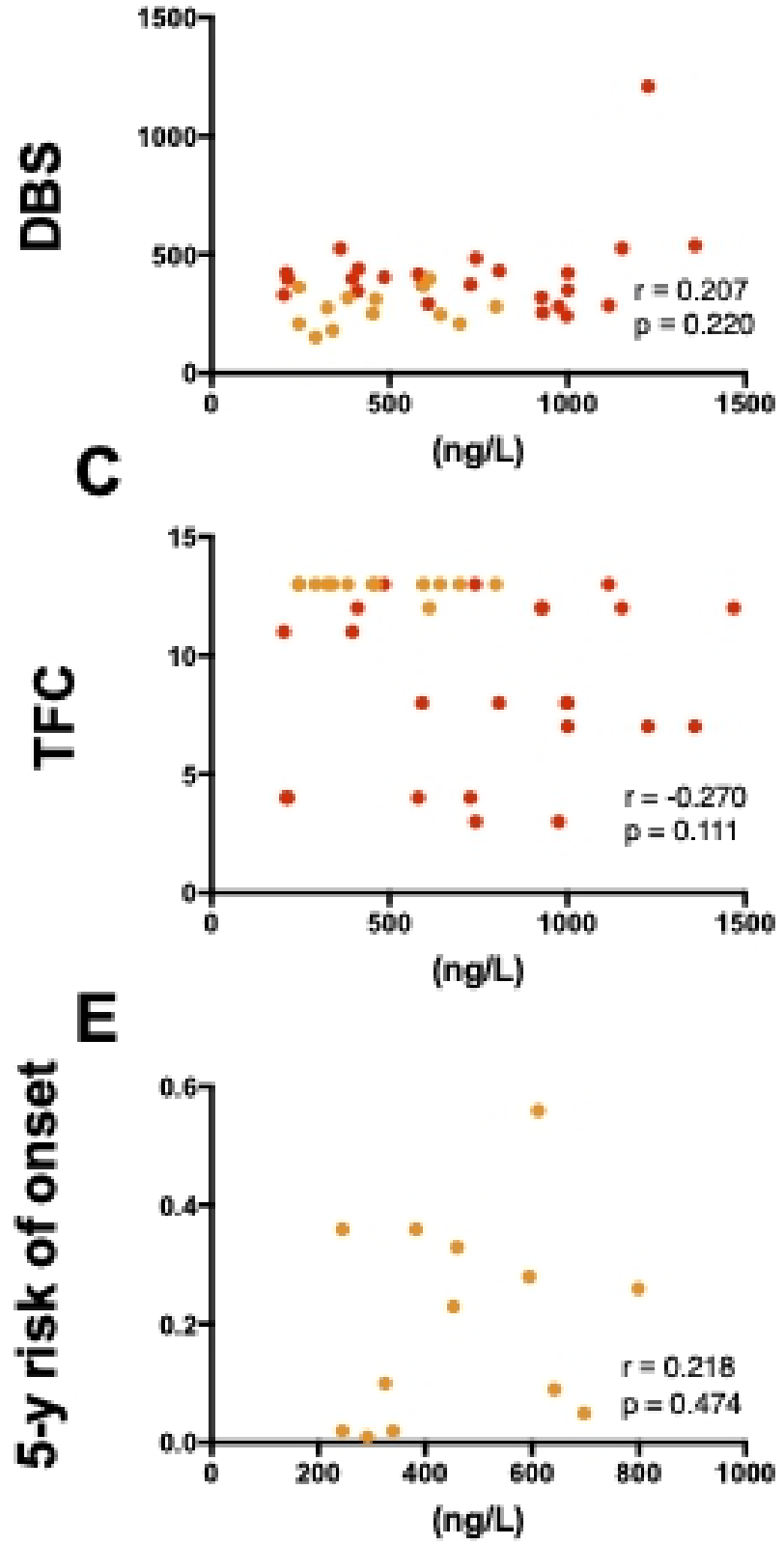

- PreHD

\section{GFAP}

B
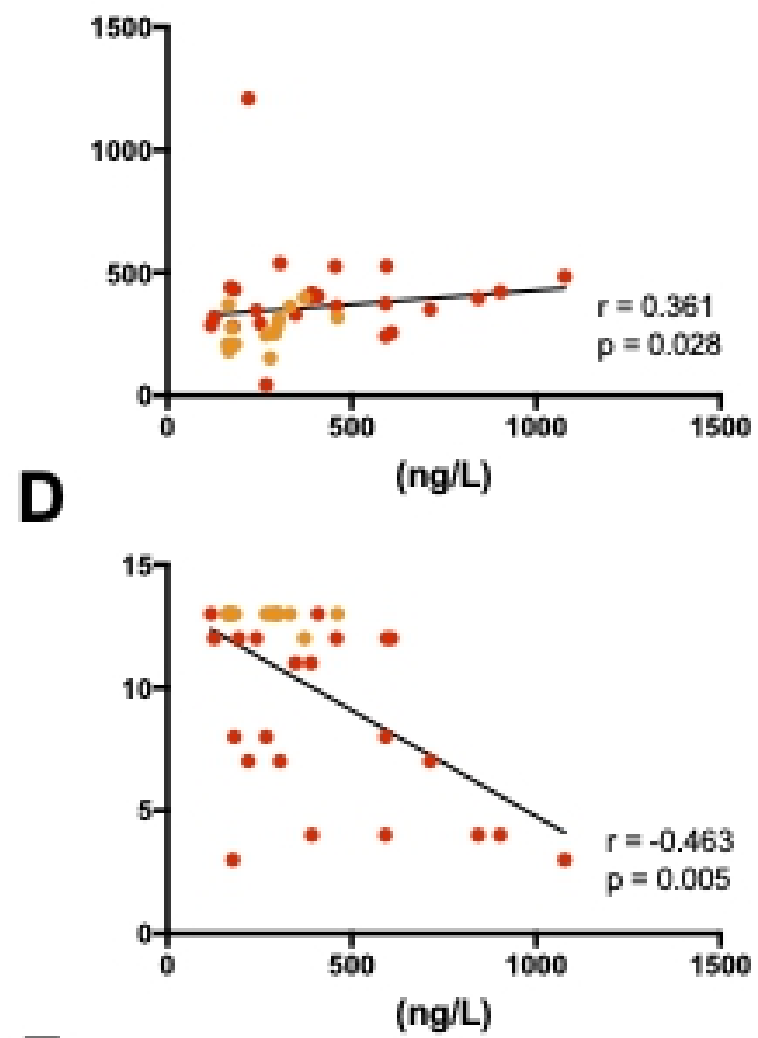

$F$

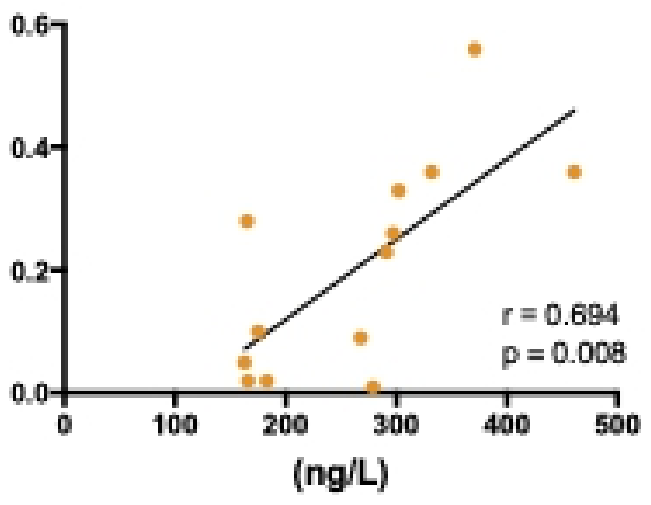

- ManHD

\section{Figure 2}

